\title{
Non-Faradaic Current Suppression in DNA Based Electrochemical Assays with a Differential Potentiostat
}

\author{
Mark D. Holtan, Subramaniam Somasundaram, Niamat Khuda, and Christopher J. Easley* \\ Department of Chemistry and Biochemistry, Auburn University, Auburn, Alabama 36849, United States
}

\begin{abstract}
One of the key factors limiting sensitivity in many electrochemical assays is the non-faradaic or capacitive current. This is particularly true in modern assay systems based on DNA monolayers at gold electrode surfaces, which have shown great promise for bioanalysis in complex milieu such as whole blood or serum. While various changes in analytical parameters, redox reporter molecules, DNA structures, probe coverage, and electrode surface area have been shown useful, background reduction by hardware subtraction has not yet been explored for these assays. Here, we introduce new electrochemistry hardware that considerably suppresses non-faradaic currents through real-time analog subtraction during current-to-voltage conversion in the potentiostat. This differential potentiostat (DiffStat) configuration is shown to suppress or remove capacitance currents in chronoamperometry, cyclic voltammetry, and square-wave voltammetry measurements applied to nucleic acid hybridization assays at the electrode surface. The DiffStat makes larger electrodes and higher sensitivity settings accessible to the user, providing order-of-magnitude improvements in sensitivity, and it also significantly simplifies data processing to extract faradaic currents in square-wave voltammetry (SWV). Since two working electrodes are used for differential measurements, unique arrangements are introduced such as converting signal-OFF assays to signal-ON assays, or background drift correction in $50 \%$ human serum. Overall, this new potentiostat design should be helpful not only in improving the sensitivity of most electrochemical assays, but it should also better support adaptation of assays to the point-of-care by circumventing complex data processing.
\end{abstract}

\section{INTRODUCTION}

Direct electrochemical analysis of living systems requires highly selective recognition and the ability to remove background interferences in matrices such as cell lysates, whole blood, or serum. Electrochemical readout has even been accomplished in vivo, although this was previously limited to enzyme-based biosensors or direct detection of endogenous electrochemically active species ${ }^{1}$. More recently, systems relying on DNA monolayers on gold electrodes for sensing or positioning of binding reagents have offered new opportunities for selective electrochemistry in complex backgrounds ${ }^{2-14}$. For example, the electrochemical DNA based (EDNA) or aptamer-based (E-AB) biosensors introduced by the Plaxco group ${ }^{15}$ have emerged as alternatives to DNA sensors or immunoassays because of their sensitivity, affordability, selectivity, and minimal instrumentation requirements ${ }^{16-20}$. Other highperformance approaches have been devised such as nanostructured electrodes $^{21}$, membrane-protected aptamers ${ }^{22}$, or antibodyoligonucleotide sensors (ECPA $)^{23-26}$, all similarly relying on DNAor RNA-based monolayers at gold electrode surfaces using standard three-electrode electrochemical cells. While these methods afford the necessary high selectivity and sensitivity for analysis of some selected analytes in living systems, there is significant room for improvement in reducing background interferences such as non-faradaic currents and signal drifts.

Optimizations of such systems have focused on instrumental parameters ${ }^{27}$, redox reporter molecules ${ }^{25,28}$, DNA structures ${ }^{12,29}$, self-assembled monolayer (SAM) coverage ${ }^{30,31}$ and chemistry ${ }^{32}$, electrode surface area ${ }^{21,33}$, and melting studies ${ }^{34,35}$. Although these are comprehensive studies, few reports have focused on reducing non-faradaic current at the electrode surface by hardware subtraction ${ }^{36}$. To our knowledge, there have been no reports exploiting hardware subtraction of non-faradaic currents with the nucleic acid-based monolayer sensors discussed above, and this presents an opportunity for further improvement in signal-to-background ratios. This non-faradaic current, which originates from the formation of a double layer at the electrode and monolayer surface, is defined by a time-dependent capacitive current in the electrochemical measurement. These currents serve as interferences (or background) to the analytical faradaic current, compromising the effectiveness of the sensors. ${ }^{37}$

One approach to reducing non-faradaic current is a working electrode surface area reduction, as the magnitude is directly proportional to surface area (SA). ${ }^{37}$ This, however, has the consequence of also reducing faradaic current, requiring higher sensitivity instrumentation. Conversely, when SA is made large, corresponding to higher currents (faradaic and non-faradaic), the instrument's amplifiers can saturate, limiting high SA electrode use, which ultimately limits the detection sensitivity of the instrument. The non-faradaic component of the electrochemical signal can also be removed during data analysis by differential measurement techniques. Indeed, differential electrochemical measurements are commonly used for noise and background reduction. ${ }^{37-40}$ Techniques such as square-wave voltammetry (SWV) or pulse voltammetry are usually applied with nucleic acid-based sensing, and recent reports have even used clever fitting of chronoamperometric data to remove background ${ }^{41}$. However, these subtractions or corrections are carried out digitally; for example, currents from specific points in the voltammogram are subtracted by means of computer 
processing. ${ }^{40}$ This process affords a digital reduction in nonfaradaic current, yet these currents remain in the raw data and consequently can limit usable electrode $\mathrm{SA}$, detection range, and sensitivity.

If non-faradaic current is removed by hardware subtraction prior to measurement of the biosensor output, analysis can be fixated on the important faradaic components of the signal. Furthermore, larger SA electrodes should be accessible, allowing larger amplitude output signals with low background, thus higher sensitivities. This approach could also open new experimental possibilities such as continuous correction in complex matrices. While other groups have shown that sequential collection and subtraction of background and signal ${ }^{42-45}$ can provide some of these benefits with in vivo neurotransmitter detection, the measurements are fundamentally made at different times, restricting the noise rejection capabilities. A few reports have applied hardware or analog subtraction of background, such as the glucose monitoring by Deman et al. ${ }^{46}$ or high density CMOS devices ${ }^{36,47,48}$, yet these works are specialized or focused on integrated microelectrode devices. Realizing that hardware background subtraction could be very useful if applied to nucleic-acid monolayer based sensors (E-DNA, AB-DNA, ECPA, etc.), we set out to construct a differential potentiostat for this purpose, starting from an open source design ${ }^{49}$, which could be used with standard electrochemical equipment.

In this paper, a differential potentiostat (DiffStat) is constructed to utilize two working electrodes, where one electrode provides a signal and the second provides a background, which is subtracted from the first. The background subtraction is performed synchronously, during data collection from both electrodes by a differential operational amplifier configuration. The differential potentiostat is then applied to a DNA monolayer based sensor and validated against a standard potentiostat with several techniques such as chronoamperometry (CA), cyclic voltammetry (CV), and SWV. As envisioned, this instrument shows improvements in surface area accessibility, increased signal, decreased background, and decreased noise. Finally, we show unique applications such as converting a traditionally "signal-off" assay to a "signal-on" assay, as well as real-time background correction in human serum.

\section{RESULTS AND DISCUSSION}

Recent advances in bioanalytical electrochemistryparticularly those built around DNA monolayers on gold electrodes-have reinvigorated the prospect of selective electrochemistry in complex matrices ${ }^{2-14}$. However, there is no obvious consensus on how to further minimize background interferences such as non-faradaic currents and signal drifts. While various clever approaches in data analysis have been devised, one option that has yet to be explored for these nucleic acid-based monolayer sensors is to carry out hardware subtraction of non-faradaic current, i.e. the capacitive current. Capacitive current generally acts as a non-zero baseline, which can place an upper limit on signal-to-noise ratios and result in narrow detection ranges. In this work, we evaluate a novel differential potentiostat (DiffStat) design (Fig. 1B) which analog subtracts much of the capacitive current within the potentiostat circuitry, outputting a signal that is predominantly composed of faradaic current. The results herein show the DiffStat to be useful for significant background reduction and reduced noise while
A)

Conventional
Potentiostat

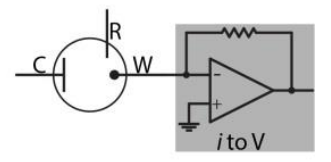

C)

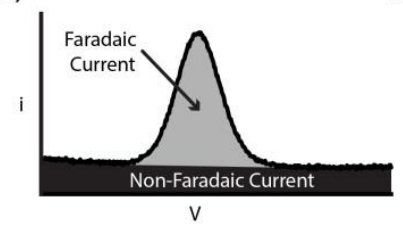

B) Differential Potentiostat

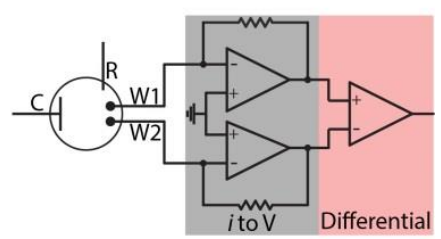

D)

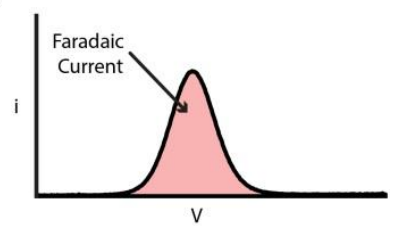

Figure 1. Comparison of a conventional potentiostat (ConStat) with the differential potentiostat (DiffStat) presented herein. Both instruments incorporate Counter, Reference and Working electrodes. A) In the ConStat, current generated by redox active molecules is measured and converted to a voltage proportional to the current. B) The DiffStat contains a second working electrode, W2, usually to serve as a non-faradaic "blank" or control electrode that is not exposed to the redox molecule. Both signal (faradaic) and baseline (non-faradaic) currents can be subtracted by a differential amplifier, yielding a corrected output (W1 - W2). C) Example data shows that the ConStat signal contains both faradaic and non-faradaic currents, while D) the DiffStat significantly reduces non-faradaic current yet preserves the faradaic current.

also supporting a wider range of electrode areas and instrument sensitivity settings.

Differential Potentiostat (DiffStat) Concept. In our attempts to address this unfavorable background at its source, we surmised that a logical way to remove the non-faradaic, capacitive currents from nucleic acid-based monolayer sensors would be to actively subtract the baseline during the measurement. One way to do this would be to build a differential circuit into the potentiostat that continuously subtracts baseline at a "blank" working electrode (W2, with no analyte) from the experimental working electrode (W1, with analyte). This would necessarily involve instrument design changes-perhaps a reason the approach has not been previously reported-yet the concept is fairly straightforward and easy to implement once the hardware changes are made. Fig. 1A shows a conventional potentiostat (ConStat) configuration using a standard cell with one working electrode connected to a current-tovoltage converter or transimpedence amplifier (TIA). By comparison, the DiffStat circuitry (Fig. 1B) utilizes a cell with two working electrodes (W1 and W2) and matching TIA circuits that feed to an additional on-board differential instrumentation amplifier. The signals from W1 and W2 are collected simultaneously, analog subtracted by the instrumentation amplifier, and quantified. By treating W1 and W2 identically, except exposing the signaling component only to $\mathrm{W} 1$, the capacitance devoid output can be generated. Example SWV signals from a surface hybridization based DNA sensor collected with the ConStat (Fig. 1C) and DiffStat (Fig. 1D) exemplify the non-faradaic current suppression capability of 


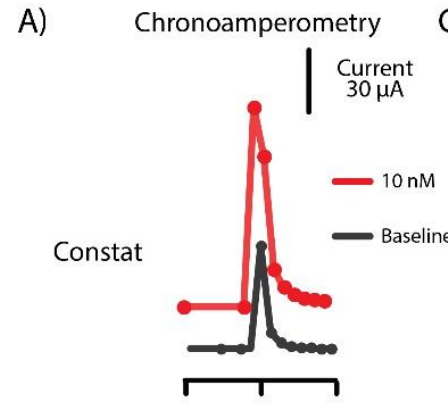

B)

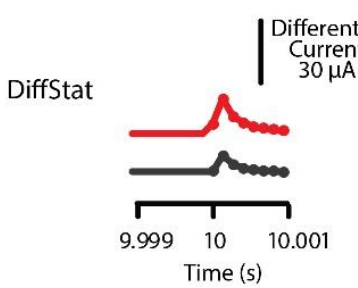

D)
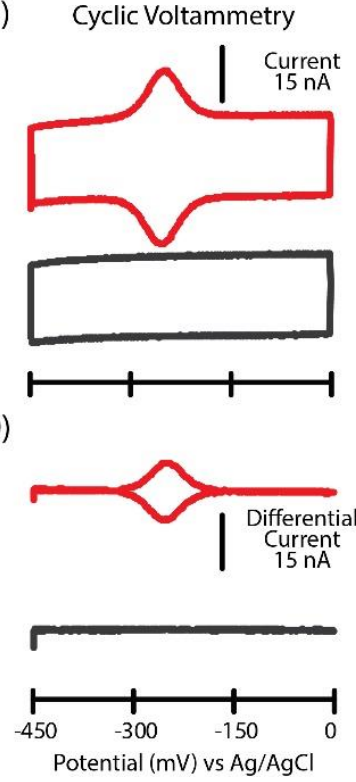

Figure 2. Classical electrochemical experiments. A) Chronoamperometry (CA) measurents from the ConStat showed higher capacitance, while B) the DiffStat CA data showed capacitance to be suppressed. C) Cyclic voltammetry (CV) by the ConStat gave $\sim 25 \mathrm{nA}$ of non-faradaic current, while $\mathrm{D}$ ) CV with the DiffStat showed efficient removal of capacitance current without losing faradaic current.

the DiffStat. While faradaic current was unchanged, non-faradaic current was greatly reduced, essentially to zero.

After initial Diffstat verification by dummy cell studies (data not shown), the DiffStat and ConStat were compared through a hybridization-based sensor for nucleic acid detection. Methylene blue (MB) appended DNA, which hybridizes to surface thiolated DNA through $40 \mathrm{bp}$, was selected as the target analyte (MB-DNA) and added to W1. The same DNA sequence in the absence of MB (CTR-DNA) was used as a control and added to W2 to carefully match the non-faradaic components between $\mathrm{W} 1$ and W2. To eliminate cross contamination between working electrodes, W1 and W2 were fabricated in two separate cells, and a split reference electrode and counter electrode were exploited for electrochemical contact (Fig. S-2). Comparative DiffStat and ConStat results using $\mathrm{CA}, \mathrm{CV}$, and $\mathrm{SWV}$ are discussed in detail below.

Chronoamperometry and Cyclic Voltammetry. Initially, the DiffStat was tested with classic techniques such as CA and CV. In $\mathrm{CA}$, the potential is quickly swept across the redox potential and held at a certain point, and current is continuously measured and plotted against the time. Fig. 2A-B shows the raw output of baseline (no analyte) and $10 \mathrm{nM}$ MB-DNA from the ConStat and DiffStat. The DiffStat showed $\sim 5$-fold suppression in capacitance current of the baseline, and a similar trend was observed with 10 nM MB-DNA. While this experiment required a detection range setting of $\pm 500 \mu \mathrm{A}$ with the ConStat, the DiffStat permitted use of the $\pm 50 \mu \mathrm{A}$ range setting (10-fold higher sensitivity) due to its baseline reduction capability.

Using CV, the current measurement is done as voltage is swept at a constant rate across the redox potential of the analyte then cycled back to the initial potential. The cyclic voltammagram outputs from the ConStat and DiffStat are shown in Fig. 2C-D. Here, the $25 \mathrm{nA}$ non-faradaic current observed by the ConStat was suppressed $99.4 \%$ by the DiffStat ( 160-fold). Conversely, the faradaic current generated by $10 \mathrm{nM}$ MB-DNA was of similar magnitude with either potentiostat. Again, an added bonus is that the DiffStat was capable of measuring at a higher instrument sensitivity range $( \pm 50 \mathrm{nA}$ and $\pm 15 \mathrm{nA}$ were used for ConStat and DiffStat, respectively). These data supported our hypothesis that the DiffStat circuitry could essentially negate non-faradaic current while preserving the faradaic current.

Square-Wave Voltammetry. DNA based electrochemical sensors have been predominantly analyzed with SWV. This is a pulse voltammetry technique developed to improve the signal-to-noise ratios, where a voltage pulse is applied, and the current measurement is delayed ( $\sim$ ms range) until capacitance current has significantly decayed ${ }^{50}$. The approach not only gives a general increase in signal-to-background ratio, but frequency-resolved SWV has also been shown capable of discriminating target-bound probes from unbound probes ${ }^{51}$ in DNA based electrochemical sensors. It was therefore important for us to thoroughly characterize the suitability of the DiffStat for SWV readout of these sensors. We carefully evaluated the system using a wide range of electrode sizes (diameters from 0.1 to $6.0 \mathrm{~mm}$ ). While large surface area electrodes are known to give proportionally higher faradaic signals, they also in-
A)

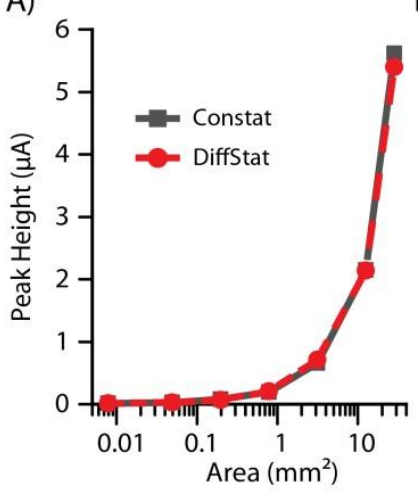

C)

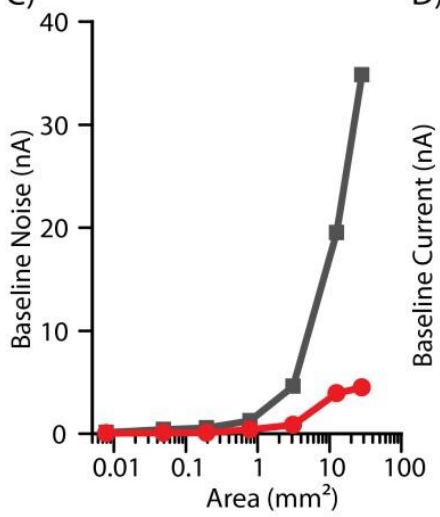

B)

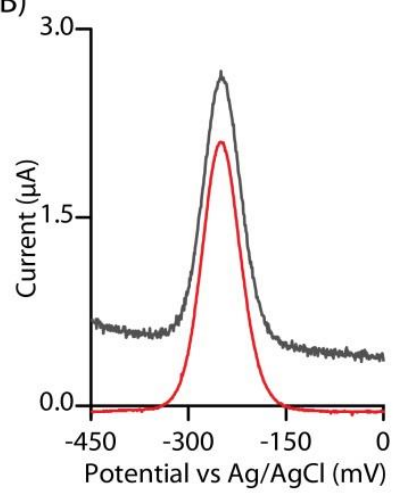

D)

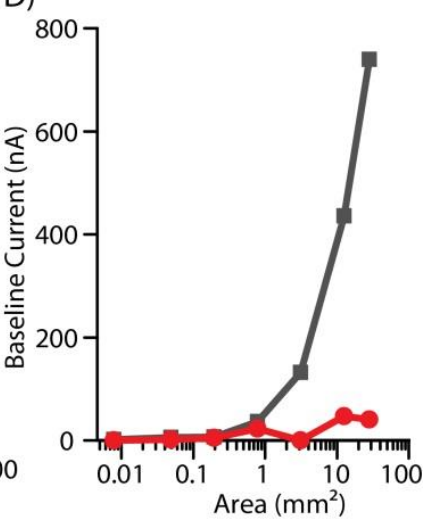

Figure 3. Non-faradaic (capacitance) suppression across a wide range of electrode areas using square-wave voltammetry (SWV). A) Faradaic peak heights from $10 \mathrm{nM}$ MB-DNA were equal using the DiffStat and ConStat, as shown in B) example unprocessed SWV data with a $4 \mathrm{~mm}$ diameter electrode. However, C) baseline noise and $\mathrm{D}$ ) non-faradaic baseline current was greatly reduced using the DiffStat (red) compared to the ConStat (gray). 
crease non-faradaic currents detrimentally, since double layer capacitance is proportional to the metal electrode's surface area. ${ }^{37}$ Large electrodes are not commonly used for this reason, but we hypothesized that the DiffStat would make larger electrodes more accessible to SWV measurements by cancelling the capacitance currents.

As expected, when sensing $10 \mathrm{nM}$ of MB-DNA (40-bp hybridization), the increases in electrode surface area gave proportional increases in both faradaic peak current (Fig. 3A, gray) and nonfaradaic baseline current (Fig. 3D, gray) with the ConStat. However, while the DiffStat's faradaic current (Fig. 3A, red) followed the same trend as the ConStat, the baseline non-faradaic current (Fig. 3D, red) was greatly suppressed. For example, the $6 \mathrm{~mm}$ electrode gave $\sim 5500 \mathrm{nA}$ of faradaic peak current and $\sim 700 \mathrm{nA}$ of non-faradaic baseline when using the ConStat; while the DiffStat showed equal peak height $(\sim 5500 \mathrm{nA})$ but only $\sim 50 \mathrm{nA}$ of nonfaradaic baseline. The SWV baseline was thus reduced $\sim 14$-fold using the DiffStat.

Additionally, the baseline noise increased proportionally with the electrode area using the ConStat (Fig. 3C, gray), likely a result of thermal noise created by the gain resistor in the potentiostat. Since the differential instrumentation amplifier in the DiffStat combats environmental noise (including thermal) by subtracting the common mode noise (noise present on both input terminals to the amplifier) during the analysis, the overall noise floor for the DiffStat instrument was lower (Fig. 3C, red). With the $6 \mathrm{~mm}$ diameter electrode, an $\sim 8$-fold reduction in noise was observed with the DiffStat compared to the ConStat.

The example square-wave voltammagrams in Fig. 3B, using the $4 \mathrm{~mm}$ diameter electrode, are representative of these findings. The ConStat data (gray) exhibited higher baseline current, higher baseline noise, and equal faradaic peak current when compared to the DiffStat data (red). These improvements given by the DiffStat contribute to better signal-to-noise ratios and effective usage of lower current ranges (higher sensitivities).

Improved Detection Range or Sensitivity in SWV. To gain a better understanding of the sensitivity improvements, we again studied the MB-DNA hybridization sensor using various electrode surface areas, where the measurements were done with 6 detection ranges (between $\pm 500 \mu \mathrm{A}$ and $\pm 15 \mathrm{nA}$ ). Note that lower detection range equates to higher sensitivity in the current-to-voltage conversion. Fig. 4 shows the comparison, where the operational ranges of the DiffStat and ConStat are plotted against surface area. At all electrode areas evaluated, these data show that DiffStat enables higher sensitivity ranges (pink/red) compared to the ConStat (gray). The labeled example in Fig. 4, using 2 mm diameter electrodes (3.14 $\mathrm{mm}^{2}$ area), gave a 10 -fold improvement in sensitivity compared to the ConStat. The DiffStat also allowed a 16-fold increase in accessible electrode area at the $1 \mu \mathrm{A}$ detection range, permitting use of the $2 \mathrm{~mm}$ diameter electrodes under that setting. The ConStat only functioned with $0.5 \mathrm{~mm}$ diameter electrodes $\left(0.196 \mathrm{~mm}^{2}\right.$ area) in the $1 \mu \mathrm{A}$ detection range, with signal saturating the instrument's analog-to-digital converter when using larger electrodes. These significant improvements in SWV sensitivity or detection range result from the strong suppression of capacitive (nonfaradaic) baseline currents by the DiffStat. While there were only 6 detection ranges available for testing in these instruments, generally

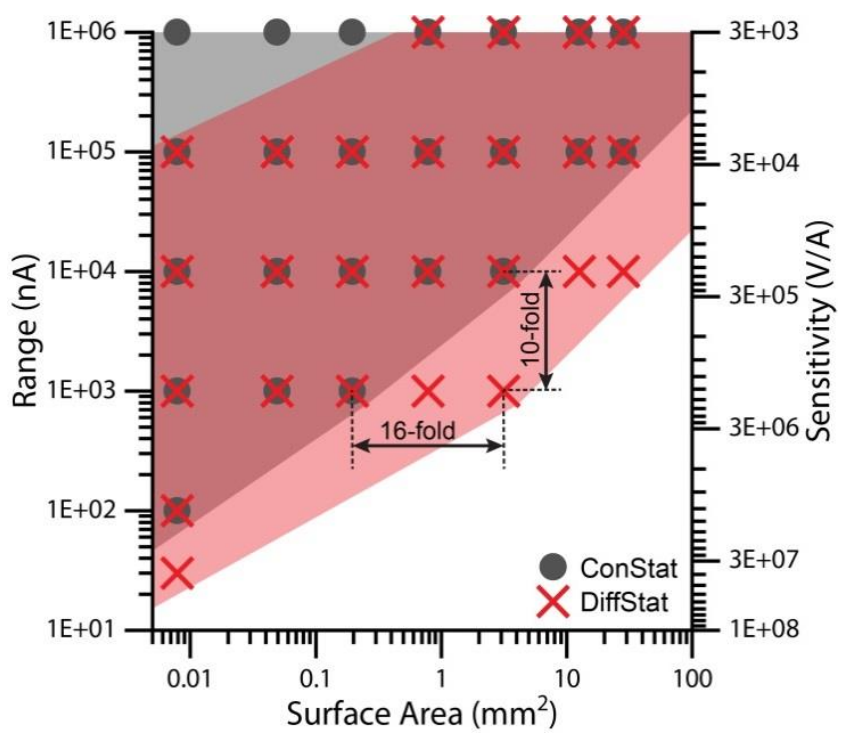

Figure 4. Detection range and sensitivity can be improved significantly with the DiffStat. Larger electrodes and more sensitive amplifier settings were accessible using the DiffStat (pink shading, red X data), settings which caused instrument overload on the ConStat (gray shading, gray circles). Data points in the figure mark instrument settings which were accessible to a given instrument.

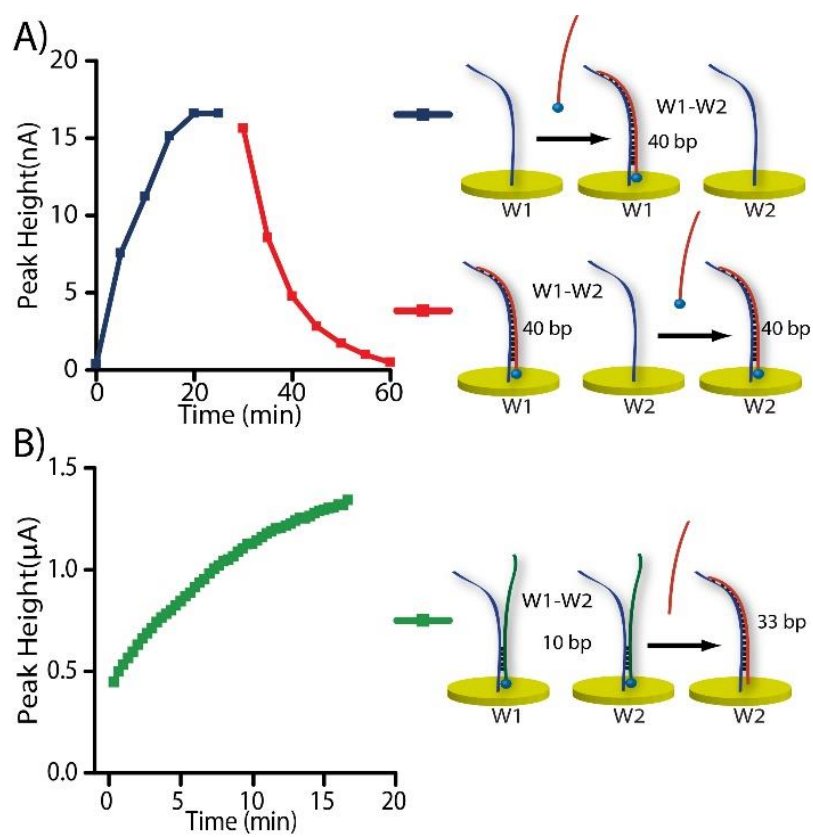

Figure 5. Unique features of the DiffStat instrument that should prove useful in bioanalysis. A) Faradaic current cancellation was shown possible by adding labeled analyte to both $\mathrm{W} 1$ and $\mathrm{W} 2$, sequentially. The initial faradaic peak increase at W1 (blue) was cancelled by adding MB-DNA also to W2, thereby subtracting the current back to zero (red) in real time. B) A signal-OFF assay could be transformed to a signal-ON assay using a similar concept, with appropriate choice of W2.

the DiffStat was shown to be capable of detecting with at least 10fold higher sensitivity than a conventional instrument, which 
should translate to lower concentrations of analytes at higher current resolution.

Faradaic Current Cancellation. Modern methods such as EDNA and aptasensors use redox molecule-appended DNA for quantification. ${ }^{52}$ In addition to the removal of capacitance current, if the initial "blank" faradaic current were also cancelled, the instrument could be used in a higher sensitivity range, which should help improve the assay ranges and LODs. Because the DiffStat's fundamental property is to subtract signal between two electrodes

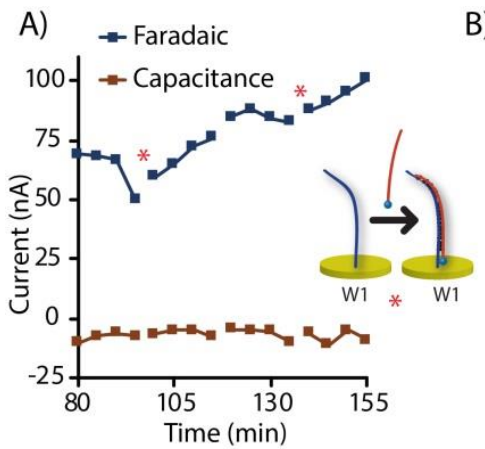

B)

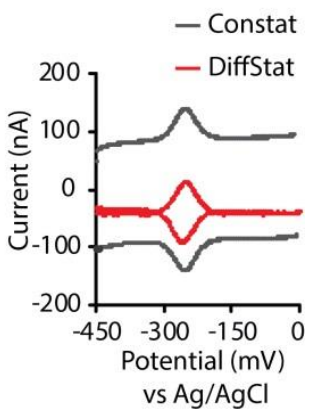

Figure 6. Signal acquisition in $50 \%$ human serum with the DiffStat. A) Over time, MB-DNA introduced in $50 \%$ human serum showed increased faradaic current with stable, near-zero non-faradaic (capacitance) current. B) CV comparisons using the DiffStat and ConStat in $50 \%$ human serum showed large nonfaradaic current with the ConStat which was absent with the DiffStat, while faradaic currents were comparable.

(W1 and W2 in Fig. 1), we hypothesized that the instrument could be used to cancel initial blank currents in sensors that start with significant faradaic signals. The data in Fig. 5A supports this hypothesis. Using a DNA hybridization sensor, MB-DNA (10 nM) was added to $\mathrm{W} 1$, while $\mathrm{W} 2$ contained no faradaic signal. As expected, the DiffStat's faradaic peak height (blue) increased over time as the MB-DNA hybridized. After it reached equilibrium ( $25 \mathrm{~min})$, the same MB-DNA (10 nM) was then added to W2. This introduced the same redox molecule to both $\mathrm{W} 1$ and $\mathrm{W} 2$, and the result was a suppression of faradaic current (red) in the final DiffStat output, which reached zero at $\sim 60 \mathrm{~min}$.

Changing Signal-OFF to Signal-ON. For a sensitive "signalOFF" assay-one that decreases signal with analyte addition - the initial signal should be large. However, to measure a larger signal, the instrument must be operated at higher current range, i.e. at lower sensitivity. Since faradaic current at W1 can be corrected with an appropriately prepared W2, the DiffStat should be capable of transforming a signal-OFF assay to a signal-ON assay, thereby allowing low concentrations to be interrogated at the instrument's highest sensitivity settings. This concept is illustrated by data in Fig. 5B, where a 33-bp competitor DNA (Com-33) was used to push pre-hybridized MB-DNA2 away from W2, thereby increasing the difference between $\mathrm{W} 1$ and $\mathrm{W} 2$. The assay which would normally be signal-OFF, was thereby transformed to a signal-ON assay. This approach should prove useful with a variety of recently developed aptasensors, E-DNA sensors, nanostructures, etc.
Measurements in human serum. One of the many advantages of DNA-monolayer-based electrochemical sensors is their proven compliance for measurement in human serum or blood (even undiluted). Nonetheless, baseline drifts remain an issue that must be dealt with in serum or plasma. Clever approaches to overcome this drift, such as measuring multiple SWV frequencies or tagging different redox molecules to the sensor have been developed ${ }^{7,8}$, where signals are later corrected during data processing. The DiffStat, however, provides a straightforward solution to address this drift by exploiting real-time subtraction of the "blank" working electrode (W2). On engaging both working electrodes into a complex matrix, the drift should be largely cancelled. Real-time DNA detection in $50 \%$ serum, as shown in Fig. 6A, validates the use of the DiffStat in complex matrices. In this experiment, every $20 \mathrm{~min}$ the $\mathrm{W} 1$ solution was alternated between $50 \%$ human serum with $10 \mathrm{nM} \mathrm{MB}$ DNA (analyte) and $50 \%$ human serum in the absence of analyte. Faradaic current was shown to increase in the presence of analyte, while no drift in the baseline was observed with or without analyte. In Fig. 6B, CV outputs from the ConStat and DiffStat were compared in $50 \%$ human serum. While the ConStat showed a large baseline, the baseline was absent when using the DiffStat. This characteristic of the DiffStat should make it particularly suitable for point-of-care detection, since sensitivity could be increased while post-measurement processing is minimized.

\section{CONCLUSIONS}

A new potentiostat arrangement was introduced, in which nonfaradaic current and environmental noise were suppressed or removed by analog subtraction of a "blank" working electrode. The DiffStat was proven useful for enhancing DNA based surface hybridization assays, enabling larger electrodes and higher sensitivity settings to be chosen. Unique arrangements of the two working electrodes allowed faradaic background subtraction that can transform signal-OFF assays to signal-ON assays or permit continuous background correction in human serum. This new instrument should also enhance the transition of SWV based assays to point-ofcare settings, since faradaic currents can be directly determined without further data processing.

Since two working electrodes are used with the DiffStat, a variety of other configurations could be devised for future studies. Care should be taken in modifying the working electrode surfaces, and the choice of using either one cell with two working electrodes or two separate cells will depend on the application. To elaborate, if the user desires to remove non-faradaic or capacitive current as shown in this work, the blank working electrode (W2) should be chosen to mimic the primary working electrode (W1) in all aspects other than the faradaic current. In other words, the non-faradaic currents between $\mathrm{W} 1$ and $\mathrm{W} 2$ should be matched. It is feasible that hardware based, real-time adjustment could be added to further improve this match in the future. Another possibility would be to apply the DiffStat to modern aptasensors ${ }^{16,17,41}$, with W1 containing the aptamer and W2 having a mutated aptamer or scrambled sequence that does not bind the target. The DiffStat output would thus be reduced to target-binding events only. Yet another example would be to tune the system to quantify small differences in redox mediator-dependent assays ${ }^{5,10}$. In our view, these are only a few of 
many unique arrangements that could be devised for and enabled by the DiffStat instrument.

\section{ASSOCIATED CONTENT}

\section{Supporting Information.}

This material is available free of charge via the Internet at http://pubs.acs.org.

Supporting figures and tables

\section{AUTHOR INFORMATION}

\section{Corresponding Author}

*Prof. Christopher J.Easley, chris.easley@auburn.edu

ORCID iD: https://orcid.org/0000-0002-2403-4147

\section{Funding Sources}

Support for this work was provided by the National Institutes of Health (R01 DK093810), by the National Science Foundation (CBET1403495), and by the Department of Chemistry and Biochemistry at Auburn University.

\section{Notes}

The authors declare the following competing financial interest (s): The differential potentiostat and methodology presented in this article was included patent application filed in March of 2018.

\section{ACKNOWLEDGMENT}

(Word Style "TD_Acknowledgments").

\section{ABBREVIATIONS}

GoG, gold on glass electrode; PDMS, polydimethylsiloxane; DiffStat, differential potentiostat; ConStat, conventional potentiostat; CA, chronoamperometry; CV, cyclic voltammetry; SWV, square wave voltammetry; MB-DNA, methylene blue DNA; CTRDNA, control DNA; Dstat, open source potentiostat; TIA, transimpedance amplifier;

\section{REFERENCES}

(1) Wilson, G. S.; Johnson, M. A. Chemical reviews 2008, 108, 2462-2481.

(2) Arroyo-Curras, N.; Somerson, J.; Vieira, P. A.; Ploense, K. L.; Kippin, T. E.; Plaxco, K. W. Proceedings of the National Academy of Sciences of the United States of America 2017, 114, 645-650.

(3) Bonham, A. J.; Paden, N. G.; Ricci, F.; Plaxco, K. W. The Analyst 2013, 138, 5580-5583.

(4) Ferguson, B. S.; Hoggarth, D. A.; Maliniak, D.; Ploense, K.; White, R. J.; Woodward, N.; Hsieh, K.; Bonham, A. J.; Eisenstein, M.; Kippin, T. E.; Plaxco, K. W.; Soh, H. T. Science translational medicine 2013, 5, 213ra165.

(5) Lam, B.; Das, J.; Holmes, R. D.; Live, L.; Sage, A.; Sargent, E. H.; Kelley, S. O. Nature communications 2013, 4, 2001.

(6) Lam, B.; Fang, Z.; Sargent, E. H.; Kelley, S. O. Analytical chemistry 2012, 84, 21-25.
(7) Li, H.; Arroyo-Curras, N.; Kang, D.; Ricci, F.; Plaxco, K. W. Journal of the American Chemical Society 2016, 138, 1580915812.

(8) Li, H.; Dauphin-Ducharme, P.; Ortega, G.; Plaxco, K. W. Journal of the American Chemical Society 2017, 139, 1120711213.

(9) Liu, J.; Wagan, S.; Davila Morris, M.; Taylor, J.; White, R. J. Analytical chemistry 2014, 86, 11417-11424.

(10) Mahshid, S. S.; Ricci, F.; Kelley, S. O.; Vallee-Belisle, A. ACS sensors 2017, 2, 718-723.

(11) Schoukroun-Barnes, L. R.; Wagan, S.; White, R. J. Analytical chemistry 2014, 86, 1131-1137.

(12) Somasundaram, S.; Easley, C. J. Journal of the American Chemical Society 2019, 141, 11721-11726.

(13) Somerson, J.; Plaxco, K. W. Molecules (Basel, Switzerland) 2018, 23.

(14) Vallee-Belisle, A.; Ricci, F.; Uzawa, T.; Xia, F.; Plaxco, K. W. Journal of the American Chemical Society 2012, 134, 1519715200.

(15) Fan, C.; Plaxco, K. W.; Heeger, A. J. Proceedings of the National Academy of Sciences of the United States of America 2003, 100, 9134-9137.

(16) Liu, Y.; Tuleouva, N.; Ramanculov, E.; Revzin, A. Analytical chemistry 2010, 82, 8131-8136.

(17) Lubin, A. A.; Plaxco, K. W. Accounts of chemical research 2010, 43, 496-505.

(18) Mahshid, S. S.; Camire, S.; Ricci, F.; Vallee-Belisle, A. Journal of the American Chemical Society 2015, 137, 1559615599.

(19) Ricci, F.; Plaxco, K. W. Microchim. Acta 2008, 163, 149155.

(20) Xiao, Y.; Piorek, B. D.; Plaxco, K. W.; Heeger, A. J. Journal of the American Chemical Society 2005, 127, 17990-17991.

(21) Soleymani, L.; Fang, Z.; Sargent, E. H.; Kelley, S. O. Nature nanotechnology 2009, 4, 844-848.

(22) Santos-Cancel, M.; White, R. J. Analytical chemistry 2017, $89,5598-5604$.

(23) Hu, J.; Wang, T.; Kim, J.; Shannon, C.; Easley, C. J. Journal of the American Chemical Society 2012, 134, 7066-7072.

(24) Hu, J.; Yu, Y.; Brooks, J. C.; Godwin, L. A.; Somasundaram, S.; Torabinejad, F.; Kim, J.; Shannon, C.; Easley, C. J. Journal of the American Chemical Society 2014, 136, 8467-8474.

(25) Ren, K.; Wu, J.; Yan, F.; Ju, H. Scientific reports 2014, 4, 4360 .

(26) Zhu, J.; Gan, H.; Wu, J.; Ju, H. Analytical chemistry 2018, 90, 5503-5508.

(27) Dauphin-Ducharme, P.; Plaxco, K. W. Analytical chemistry 2016, 88, 11654-11662.

(28) Kang, D.; Ricci, F.; White, R. J.; Plaxco, K. W. Analytical chemistry 2016, 88, 10452-10458.

(29) Huang, K. C.; White, R. J. Journal of the American Chemical Society 2013, 135, 12808-12817. 
(30) Ricci, F.; Lai, R. Y.; Heeger, A. J.; Plaxco, K. W.; Sumner, J. J. Langmuir : the ACS journal of surfaces and colloids 2007, 23, 6827-6834.

(31) White, R. J.; Phares, N.; Lubin, A. A.; Xiao, Y.; Plaxco, K. W. Langmuir : the ACS journal of surfaces and colloids 2008, 24, 10513-10518.

(32) Li, H.; Dauphin-Ducharme, P.; Arroyo-Curras, N.; Tran, C. H.; Vieira, P. A.; Li, S.; Shin, C.; Somerson, J.; Kippin, T. E.; Plaxco, K. W. Angewandte Chemie (International ed. in English) 2017, 56, 7492-7495.

(33) Hauke, A.; Kumar, L. S. S.; Kim, M. Y.; Pegan, J.; Khine, M.; Li, H.; Plaxco, K. W.; Heikenfeld, J. Biosensors \& bioelectronics 2017, 94, 438-442.

(34) Somasundaram, S.; Holtan, M. D.; Easley, C. J. Analytical chemistry 2018, 90, 3584-3591.

(35) Yang, A. H.; Hsieh, K.; Patterson, A. S.; Ferguson, B. S.; Eisenstein, M.; Plaxco, K. W.; Soh, H. T. Angewandte Chemie (International ed. in English) 2014, 53, 3163-3167.

(36) Hassibi, A.; Lee, T. H. IEEE Sens. J. 2006, 6, 1380-1388.

(37) Bard, A. J.; Faulkner, L. R. Electrochemical Methods: Fundamentals and Applications; Wiley, 2000.

(38) Jacobs, P.; Suls, J.; Sansen, W. Sensors and Actuators B: Chemical 1994, 20, 193-198.

(39) McGrath, M. J.; Iwuoha, E. I.; Diamond, D.; Smyth, M. R. Biosensors \& bioelectronics 1995, 10, 937-943.

(40) Osteryoung, J. G.; Osteryoung, R. A. Analytical chemistry $1985,57,101-110$.

(41) Arroyo-Curras, N.; Dauphin-Ducharme, P.; Ortega, G.; Ploense, K. L.; Kippin, T. E.; Plaxco, K. W. ACS sensors 2018, 3, 360-366.
(42) Dorta-Quiñones, C. I.; Wang, X. Y.; Dokania, R. K.; Gailey, A.; Lindau, M.; Apsel, A. B. IEEE Transactions on Biomedical Circuits and Systems 2016, 10, 289-299.

(43) Hermans, A.; Keithley, R. B.; Kita, J. M.; Sombers, L. A.; Wightman, R. M. Analytical chemistry 2008, 80, 4040-4048.

(44) Howell, J. O.; Kuhr, W. G.; Ensman, R. E.; Mark Wightman, R. Journal of Electroanalytical Chemistry and Interfacial Electrochemistry 1986, 209, 77-90.

(45) Wang, J.; Dewald, H. D. Talanta 1984, 31, 387-390.

(46) Deman, P.; Suls, J.; Sansen, W. Sensors and Actuators B: Chemical 1997, 44, 304-308.

(47) Martin, S. M.; Gebara, F. H.; Strong, T. D.; Brown, R. B. IEEE Sens. J. 2009, 9, 135-142.

(48) Nazari, M. H.; Genov, R. In 2009 IEEE International Symposium on Circuits and Systems, 2009, pp 2177-2180.

(49) Dryden, M. D.; Wheeler, A. R. PloS one 2015, 10, e0140349.

(50) Ramaley, L.; Krause, M. S. Analytical chemistry 1969, 41, 1362-1365.

(51) White, R. J.; Plaxco, K. W. Analytical chemistry 2010, 82, 73-76.

(52) Kang, D.; Zuo, X.; Yang, R.; Xia, F.; Plaxco, K. W.; White, R. J. Analytical chemistry 2009, 81, 9109-9113. 